\title{
Impact of polyelectrolyte multilayers on the ionic current rectification of conical nanopores.
}

Tianji Ma ${ }^{1}$, Paulius Gaigalas ${ }^{1,2}$, Mathilde Lepoitevin ${ }^{1}$, Ieva Plikusiene ${ }^{2,3}$, Mikhael Bechelany ${ }^{1}$, Jean-Marc Janot $^{1}$, Emmanuel Balanzat ${ }^{4}$, and Sebastien Balme ${ }^{1 *}$

${ }^{1}$ Institut Européen des Membranes, UMR5635 UM ENSM CNRS, Place Eugène Bataillon, 34095 Montpellier cedex 5, France

${ }^{2}$ Vilnius University, Faculty of chemistry and geosciences, Naugarduko str 24, Vilnius, Lithuania

${ }^{3}$ Center for Physical Sciences and Technology, Department of material science and electrical engineering, Sauletekio av. 3, Vilnius, Lithuania

${ }^{4}$ Centre de recherche sur les Ions, les Matériaux et la Photonique, UMR6252 CEA-CNRS-ENSICAEN, 6 Boulevard du Maréchal Juin, 14050 Caen Cedex 4, France

$\dagger$ Corresponding author sebastien.balme@umontpellier.fr.

\section{Abstract :}

\section{Keywords :}

\section{Introduction}

For the last two decades, single nanopore technology has contributed to the emergence and design of nano-fluidic responsive systems $[1,2]$. It has provided a deep understanding of the transport from simple electrolyte to (bio)macromolecule (DNA, polymer or protein) at the nano-scale, contributed to the development of new analytical device for DNA sequencing. Nanopores with a dissymmetry of charge or shape are also particularly fascinating for its ionic diode [3-5]. The latter are usually obtained using conical or semi-cigare shape nanopores in polymer film such as PET, PC or PI using track-etched technique [6]. Ionic diode behavior comes from a dissymmetrical distribution of the ions inside the 
nanopore which induces a depletion of anions or cations $[7,8]$. It can be tuned by the functionalization of the surface of the nanopore walls. For PET, the carboxyl moieties are particularly interesting because they can be easy functionalized with a large range of molecules or macromolecules [9-11]. Many stimuli responsive channel such as $\mathrm{pH}[12,13]$, light $[14,15]$ have been designed using this strategy. As the current rectification is strongly dependent of the nanopore surface charge, it can be used to develop sensors, when molecules or macromolecules are attached at its surface [16, 17].

Amongst all the possibilities offered by track-etched nanopores, the ability to modify the ionic transport with $\mathrm{pH}$ is particularly interesting. It involves the grafting of functions onto the nanopore surface wall, exhibiting different charges as a function of $\mathrm{pH}$. For example, poly-protonic polymer brushes permit a modulation of the current rectification [18]. Amphoteric molecules such as lysine or histidine [19] were used to modify the nanopore selectivity by changing the $\mathrm{pH}$. At low $\mathrm{pH}$, the nanopore is positively charged and selective to anion, while at high $\mathrm{pH}$ it becomes negatively charged and thus selective to cation. Similar properties can be obtained using poly(methacryoyl-L-lysine) brushes. Spyropyrans modify the ionic transport by modifying either or both $\mathrm{pH}$ and light [14]. All those strategies are based on the chemical grafting of responsive molecules onto the carboxyl moieties.

The layer-by-layer deposition of polyelectrolyte is an interesting method to functionalize membranes and nanopores to obtain ionic diode behaviors. It allows to improve their ionic selectivity, and reduces the fouling by proteins $[20,21]$. The deposition of poly-L-lysine can inverse the current rectification. The advantage of this functionalization is its reversibility. Due to the electrostatic interactions of the electrolyte with the surface, reversible and modular sensors can be designed. Poly(allylamine hydrochloride) (PAH) poly(styrenesulfonate) (PSS) have been used to solve by a mathematical model the exact shape of nanopore [22]. Based on finite element simulation of PNP equation, the authors demonstrated that the surface charge of the pore walls decreases dramatically with the number of PAH/PSS layers assembled into the nanopore, the current rectification tend to 1. 
We assume that confined multilayers of polyelectrolytes create a zwitterionic film onto the nanopore surface. It should exhibit similar properties to the ones observed after grafting amphoteric molecules. We expect to obtain a nanopore selective to anion at low $\mathrm{pH}$ and cation at high $\mathrm{pH}$. Such behavior had been reported for a conical nanopore functionalized with chitosan/ polyacrylic acid (PAA) [23]. However, the behavior of polyelectrolytes is complex and strongly depends on the $\mathrm{pH}$, especially for the weak ones by changing ionic strength or $\mathrm{pH}$ induces swelling of the structure of the polyelectrolyte layer as well as modifying the ionic transport. This was evidenced by nanopores functionalized with polyethylenimine (PEI) and chondroitin-4-sulfate (ChS) layers[13]. The swelling of ChS which occurs at low pH induces original gating properties. The nanopore closes at $\mathrm{pH}>4$ and let only the anions go through the nanopore at $\mathrm{pH}<4$. This phenomenon only occurs at low salt concentration. Regarding the opposite behavior of these two systems, it is necessary to go further in the investigation on the ionic transport properties of the conical nanopore functionalized by polyelectrolyte multi-layer.

In this work, we have functionalized single conical nanopore in PET, obtained by track-etched technique with different couples of polyelectrolytes: poly-L-lysine (PLL) /PAA and PEI/PAA. The influence of pH and divalent cation is reported. Spectroscopic ellipsometry experiments were performed on surfaces to know the influence of salt and $\mathrm{pH}$ on the thickness of the multilayers of polyelectrolytes. Finally, we also reported the impact of cross linking of several polyelectrolyte layers in order to prevent large conformational changes.

\section{Material and Methods}

\subsection{Material}

Poly(ethylene terephthalate) (PET) film (thickness $13 \mu \mathrm{m}$, biaxial orientation) was purchased from Goodfellow (ES301061). Sodium chloride (71380), potassium chloride (P3911), sodium hydroxide (30603), hydrogen chloride (30721), branched PEI (Mw 25 kD, 408727), PAA (Mw 100 kD, 523925), PLL (30 kD-60kD P2636) were purchased from Sigma-Aldrich. Ultra-pure water was produced from a Q$\operatorname{grad}{ }^{\circledR}-1$ Milli-Q system (Millipore). 


\subsection{Track-etching nanopores and characterization}

Single tracks were produced by Xe irradiation $(8.98 \mathrm{MeV})$ in PET films $(13 \mu \mathrm{m})$ (GANIL, SEM line, Caen, France). The PET films were exposed to UV light for $24 \mathrm{~h}$ per side to activate the track (Fisher bioblock; VL215.MC, $\lambda=302 \mathrm{~nm}$ ), before the chemical etching process. The etching of the conical nanopore was performed under dissymmetric condition following the electro-stopping method.[5] The PET foil was mounted between the two compartments of a chemical cell in Teflon. The etchant solution $(\mathrm{NaOH} 9 \mathrm{M}, 1.6 \mathrm{ml})$ was added on the base side and the stopping solution $(\mathrm{KCl} 1 \mathrm{M}$ and acetic acid $1 \mathrm{M}$, $1.6 \mathrm{ml}$ ) on the tip side. A potential of $1 \mathrm{~V}$ was applied across the membrane; the reference electrode is immersed in the stop solution and the working one in the etchant solution. When the current reaches a value of several hundred $\mathrm{pA}$, the etching process was stopped by the replacement of the etching solution by the stop one.

The conical nanopores are characterized as follow. The base diameter $(D)$ is calculated as a function of the total etch time $t(\mathrm{~min})$ using the relationship determined in our lab $D=2.5 t$. - The tip diameter ( $d$ ) was calculated, assuming a bulk-like ionic conductivity inside the nanopores, from the dependence of the conductance $G$ to the $\mathrm{NaCl}$ concentration (equation 1).[24] In order to correct the conductivity at high salt concentration, the diameter is calculated using the ionic conductivity of solution $\kappa$. The latter have been measured using a conductimeter (Hanna HI 255 combined meter with conductivity and electrode HI 76310) after preparation.

$G=\kappa \pi D d / L(1)$

where $L$ is the nanopore length.

\subsection{Current-voltage measurements}

Electrical measurements were performed using a patch-clamp amplifier (EPC10 HEKA electronics, Germany). The current is measured by $\mathrm{Ag} / \mathrm{AgCl}$ electrode. A single nanopore was mounted in the 
chemical Teflon cell containing an electrolyte solution. One electrode was plugged to the working end of the amplifier (trans chamber, base side) and the other electrode connected to the ground (cis chamber, tip side). Recorded currents were analyzed by Fitmaster (Heka Elektronik, Germany).

For I-V curves, the currents data were recorded as a function of the time under constant voltage from -1 V to $1 \mathrm{~V}$ by $100 \mathrm{mV}$ step and from $-100 \mathrm{mV}$ to $100 \mathrm{mV}$ by $10 \mathrm{mV}$ steps. All current traces were recorded during $10 \mathrm{~s}$ at a frequency of $50 \mathrm{kHz}$. These measurements were performed 3 times. The conductance $G$ is extracted from the linear zone of I-V curves from typically - $75 \mathrm{mV}$ to $75 \mathrm{mV}$.

\subsection{Nanopore functionalization}

PAA, PEI and PLL stock solutions at concentration of $1 \mathrm{mg} \mathrm{ml}^{-1}, \mathrm{pH} 7$ were prepared. The functionalization of the nanopores was performed under $\mathrm{KCl} 0.1 \mathrm{M} \mathrm{pH} 7$ by addition of $16 \mu$ of stock solution at the tip side. $-1 \mathrm{~V}$ was applied for PEI and PLL during 5 minutes. Then the cell was filled with buffer to remove the excess of polyelectrolytes. Then $16 \mu$ l of PAA solution was added at the tip side during 5 minutes applying $1 \mathrm{~V}$. The procedure is repeated until the desired numbers of PEI/PAA or PLL/PAA bilayers. During the deposition, the current is recorded at a $5 \mathrm{kHz}$ frequency in order to monitor the adsorption of the polyelectrolytes.

Table 1. list of the nanopores used in this work

\begin{tabular}{|l|l|l|l|l|l|}
\hline Nanopore & $\mathrm{d}$ & $\mathrm{D}$ & ${\text { (Polyelectrolyte })_{\text {\#layers }}}$ & & Code \\
\hline NP-1 & 30 & 300 & $(\mathrm{PLL})_{3} /(\mathrm{PAA})_{3}$ & & 31 PET13B20 \\
\hline NP-2 & 28 & 475 & $(\mathrm{PEI})_{4} /(\mathrm{PAA})_{4}$ & & $29 \mathrm{PET} 13$ A12 \\
\hline NP-3 & 116 & 346 & $(\mathrm{PLL})_{10} /(\mathrm{PAA})_{10}$ & & $30 \mathrm{PET} 13 \mathrm{C} 02$ \\
\hline
\end{tabular}




\begin{tabular}{|l|l|l|l|l|l|}
\hline NP-4 & 91 & 460 & $(\mathrm{PEI})_{8} /(\mathrm{PAA})_{8}$ & & $30 \mathrm{PET} 13 \mathrm{C} 04$ \\
\hline NP-5 & & & $(\mathrm{PLL})_{4} /(\mathrm{PAA})_{4}$ & crosslinked & \\
\hline
\end{tabular}

\subsection{Spectroscopic ellipsometry}

Ellipsometric investigation of PLL/PAA and PEI/PAA bilayers were performed using spectral ellipsometer from J. A. Woollam M2000X on silicon wafer substrate. Firstly surface of the wafer was activated using piranha solution $\left(\mathrm{H}_{2} \mathrm{SO}_{4}: \mathrm{H}_{2} \mathrm{O}_{2} 3: 1\right)$ to form $-\mathrm{OH}$ groups at the surface, necessary for the immobilization of the polyelectrolyte layer. Ellipsometric spectra were recorded from $200 \mathrm{~nm}$ to 1000 $\mathrm{nm}$, angle of incident light was $70^{\circ}$. Cauchy dispersion function was applied for the regression analysis of the formed PEI, PAA and PLL layers. Optical constants and thicknesses of these layers were obtained using Complete EASE software.

\section{Results and discussion}

\subsection{Polyelectrolyte deposition.}

Single conical nanopores were obtained in PET film (thickness $13 \mu \mathrm{m}$ ) by track-etched method. The nanopores used for this study are reported in Table 1. More than 8 bilayers can be deposited into conical nanopores with a large tip diameter $(d \sim 100 \mathrm{~nm})$. For small tip diameter $(d \sim 30 \mathrm{~nm})$ less than 4 bilayers was used. The nanopore internal surface is covered by carboxylate groups and thus exhibits a negative surface charge at $\mathrm{pH}$ 8. The I-V dependence recorded from $-1 \mathrm{~V}$ to $1 \mathrm{~V}$ curves show a current rectification characterized by the rectification factor $\left(f_{\text {rec }}\right)$ calculated from $f_{\text {rec }}=\left|I_{(1 V)}\right| /\left|I_{(-1 V)}\right|$. Under our experimental condition (Figure 1), the current rectification is lower than 1 when the nanopore is negatively charged. The polycation (PEI or PLL) or polyanion (PAA) deposition was performed by adding them at the tip side under a voltage of $-1 \mathrm{~V}$ or $1 \mathrm{~V}$ respectively. This voltage provides the required energy at the polyelectrolyte to overcome the barrier of entrance through nanopore. Independently of the 
polyelectrolyte nature, the current recorded as a function of the time shows a strong decrease, which is induced by the charge inversion at the nanopore entrance [21]. Indeed after the adsorption of the polycations, the nanopore becomes positively charged and thus the rectification factor increase above 1 (Figure 1), while after the adsorption of the polyanions, the nanopore becomes negatively charged and the rectification factor decrease below 1 . The inversion of the rectification factor brings evidence that each layer of polyelectrolytes has been deposited successfully. According to previous work, we can assume that the polyelectrolyte deposition occurs at the narrow opening.

The dependence of $f_{\text {rec }}$ with the number of bilayer strongly depends on the polyelectrolyte couple. For (PLL) ${ }_{10} /(\mathrm{PAA})_{10}$, it tends to a value around $10^{-3}$. When increasing the number of bilayers, the addition of PLL does not induce a clear inversion of the rectification factor. Similar behavior was previously reported in the case of $\mathrm{PEI} / \mathrm{ChS}$ deposition [13]. It was assigned to a partial charge compensation between either polyelectrolytes or an in-homogeneous surface charge patterns between the region close to the narrow opening and the rest of the nanopore. For PEI/PAA, the inversion of rectification occurs after each deposition likely due to a better charge compensation between both polyelectrolytes. However, it is interesting to note that $f_{\text {rec }}$ does not tend to 1 as reported for PAH/PSS. 
NP-3

(a)

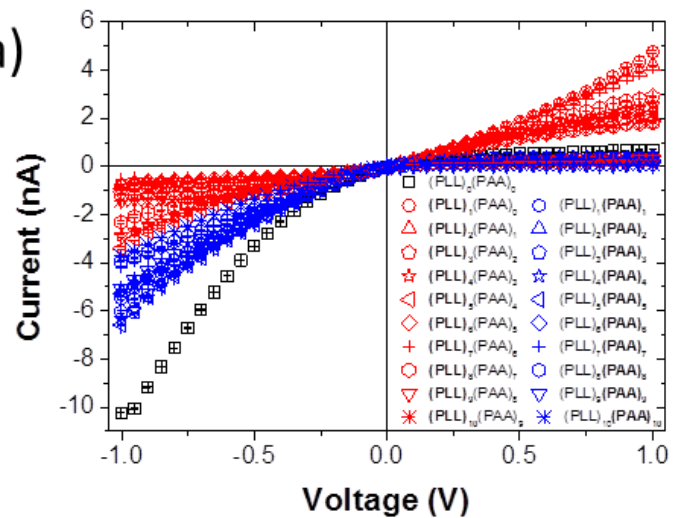

(c)

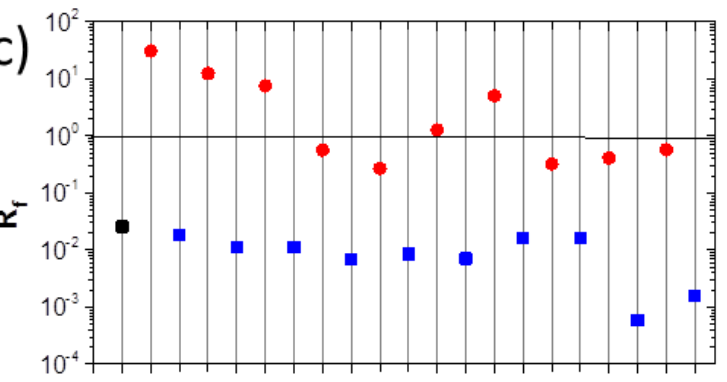

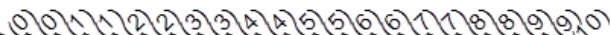
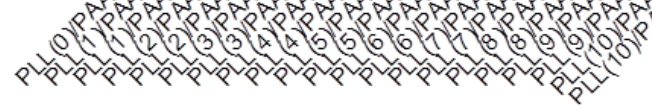

(e)

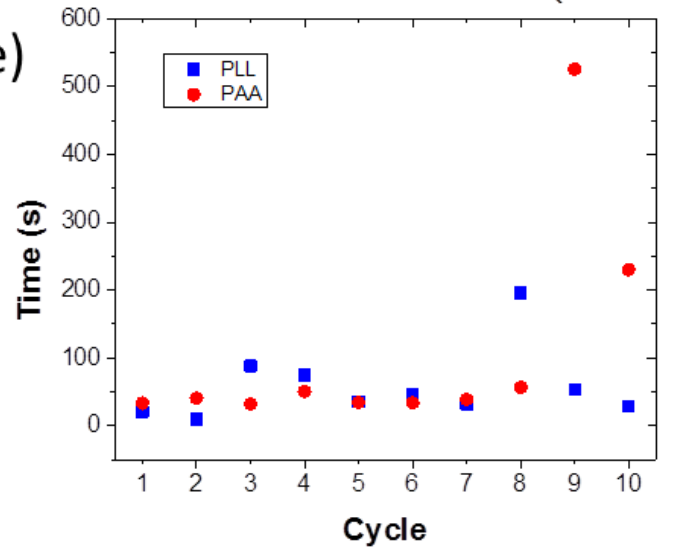

NP-4

(b)

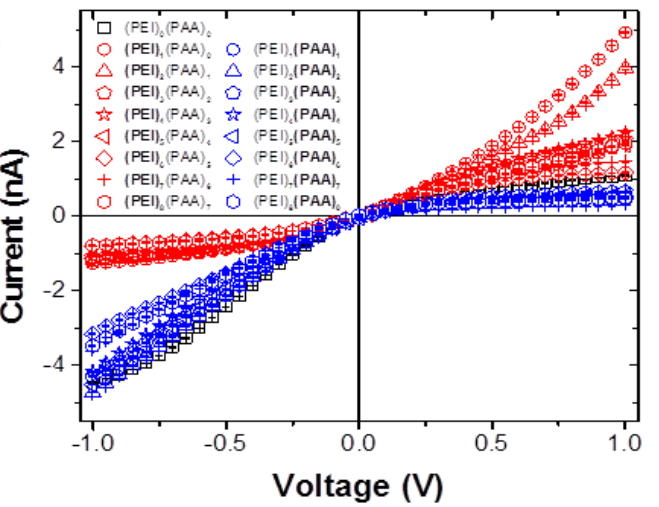

(d)
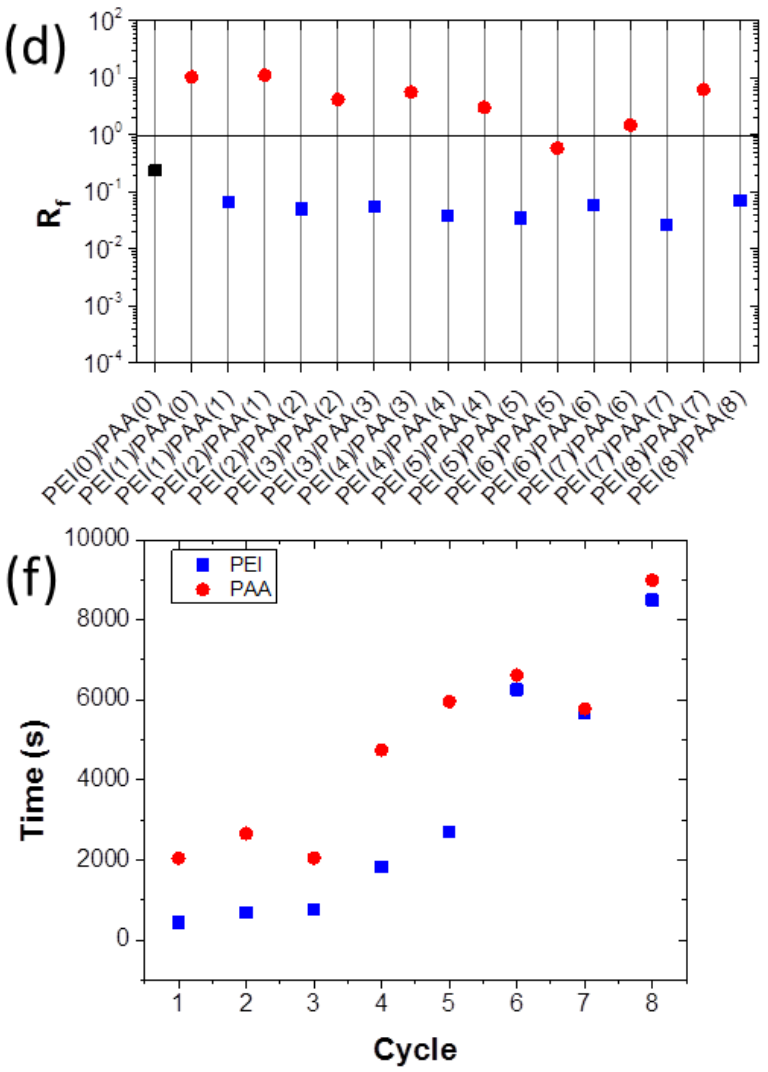

Figure 1 : characterization of the nanopore functionalization : I-V dependence after polyelectrolyte deposition recorded at $\mathrm{NaCl}$ concentration of $0.1 \mathrm{M}$ (a) NP-3 (b) NP-4. Rectification factor after the deposition of each electrolyte (c) NP-3 (d) NP-4. Time required to observe the inversion of current rectification induced by the polyelectrolyte deposition (e) NP-3 (f) NP-4

The time required for the polyelectrolyte to be deposited is very dependent on the nature of the polycation. In the case of PLL, PAA is deposited in less than $120 \mathrm{~s}$ for the 7 first bilayers and requires more time for the layers 8 to 10 probably due to steric hindrance. With PEI, the deposition of PAA 
requires more than $2000 \mathrm{~s}$. The difference of time is clearly dependent of the polycation which can influence the structure of the bilayer inside the nanopore. In order to characterize this behavior, the thickness of PLL/PAA and PEI/PAA bilayers were investigated using spectroscopic ellipsometry. The polyelectrolyte bilayers were deposited on the surface of a wafer. The obtained values of refraction index dispersion are presented in Figure 2 and thicknesses of the layers are presented in Erreur ! Source du renvoi introuvable.. Figure SI-1 of raw Psi and Delta data, shows that additional bilayers are formed on the top of the silicon substrate. The building of an optical model becomes very complicated in this case when more bilayers are deposited on the substrate. Optical model well fits data for single PLL, PEI layers and for PLL/PAA and PEI/PAA bilayers only. After fitting using Cauchy dispersion formula, refractive index dispersion and thicknesses of these layers are calculated. Spectroscopic ellipsometry characterization evidences a difference of bilayer thickness which are $7.28 \mathrm{~nm}$ and $3.91 \mathrm{~nm}$ for PEI/PAA and PLL/PAA respectively. This difference is mainly due to the thickness of the PAA layer and thus its conformation which is more folded in presence of PEI. The longer time can thus be interpreted by steric effect which leads to a more complicated penetration of the polyelectrolyte inside the nanopore and/or the necessity to add more polymer chains to cover the polycation layer. 


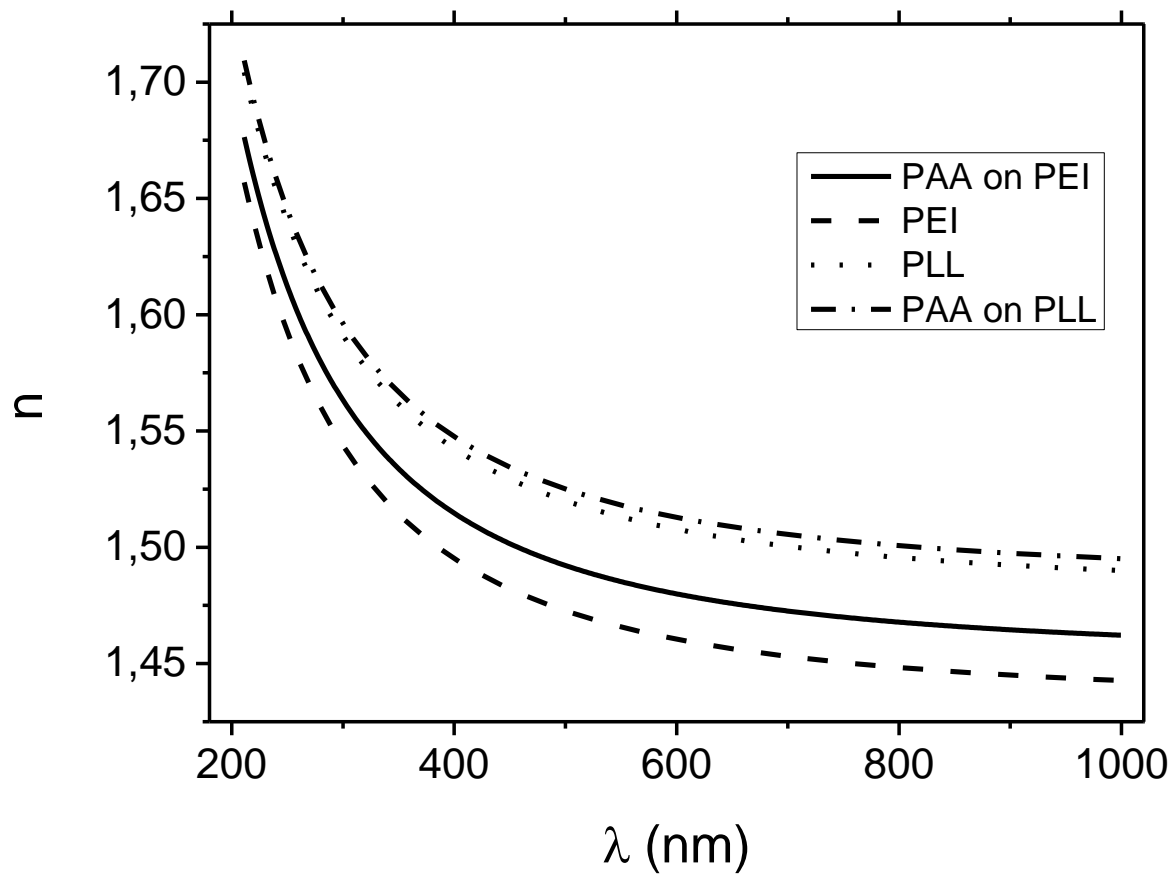

Figure 2 : Refractive indexes dispersion obtained for PAA, PEI and PLL layers deposited on silicon wafer substrate.

Table 2. Thicknesses of PLL, PAA, PEI layers on silicon wafer

\begin{tabular}{|l|l|l|l|l|}
\hline & PEI/PAA & & PLL/PAA & \\
\hline & PEI & PAA & PLL & PAA \\
\hline thickness, nm & 2.14 & 5.14 & 1.68 & 2.23 \\
\hline
\end{tabular}

\subsection{Ionic transport through nanopores modified by LbL deposition}

The ionic transport properties of the nanopore decorated with the polyelectrolyte were studied for different $\mathrm{NaCl}$ concentrations (from $10^{-3}$ until $1 \mathrm{M}$ ) at various pHs (Figure 3). Because the pKa of PAA is 4.5 , we can expect that at $\mathrm{pH} 3$ the nanopore will be globally positively charged inducing an inversion of the rectification factor. 


\subsubsection{PEI/PAA LbL functionalization of a nanopore}

In Figure 3 are reported the I-V dependence at $\mathrm{pH} 3$ and 7 for two nanopores with large and small diameters. For the large nanopore $(\mathrm{d}=91 \mathrm{~nm})$ functionalized with 8 layers of PEI/PAA, the I-V dependence shows a rectification factor below 1 at $\mathrm{pH}$ 7. Interestingly, at $\mathrm{pH} 3$, the inversion of the rectification factor occurs at low salt concentration $(\mathrm{NaCl}=0.01$ and $0.05 \mathrm{M})$. When increasing the concentration, the nanopore seems closed and the rectification factor is close to 1. $\mathrm{pH}$-gating properties under acidic conditions were previously reported for nanopore decorated with PEI/ChS [13]. However in that case, with the increase of the salt concentration, the gating property disappeared. With the PEI/PAA, the interesting result is that the nanopore is selective to anion at low salt concentration at $\mathrm{pH} 3$. To confirm this property, the same study was performed in a conical nanopore with a smaller tip diameter (29 $\mathrm{nm})$ decorated with 4 PEI/PAA bilayers. The I-V dependences reported on Figure 3 reveal the same behavior. For these two nanopores, the enclosure at $\mathrm{pH} 3$ arises for a $\mathrm{NaCl}$ concentration of $0.1 \mathrm{M}$. At $\mathrm{pH}$ 4, the nanopore seems closed independently of the $\mathrm{NaCl}$ concentration. 
NP-2
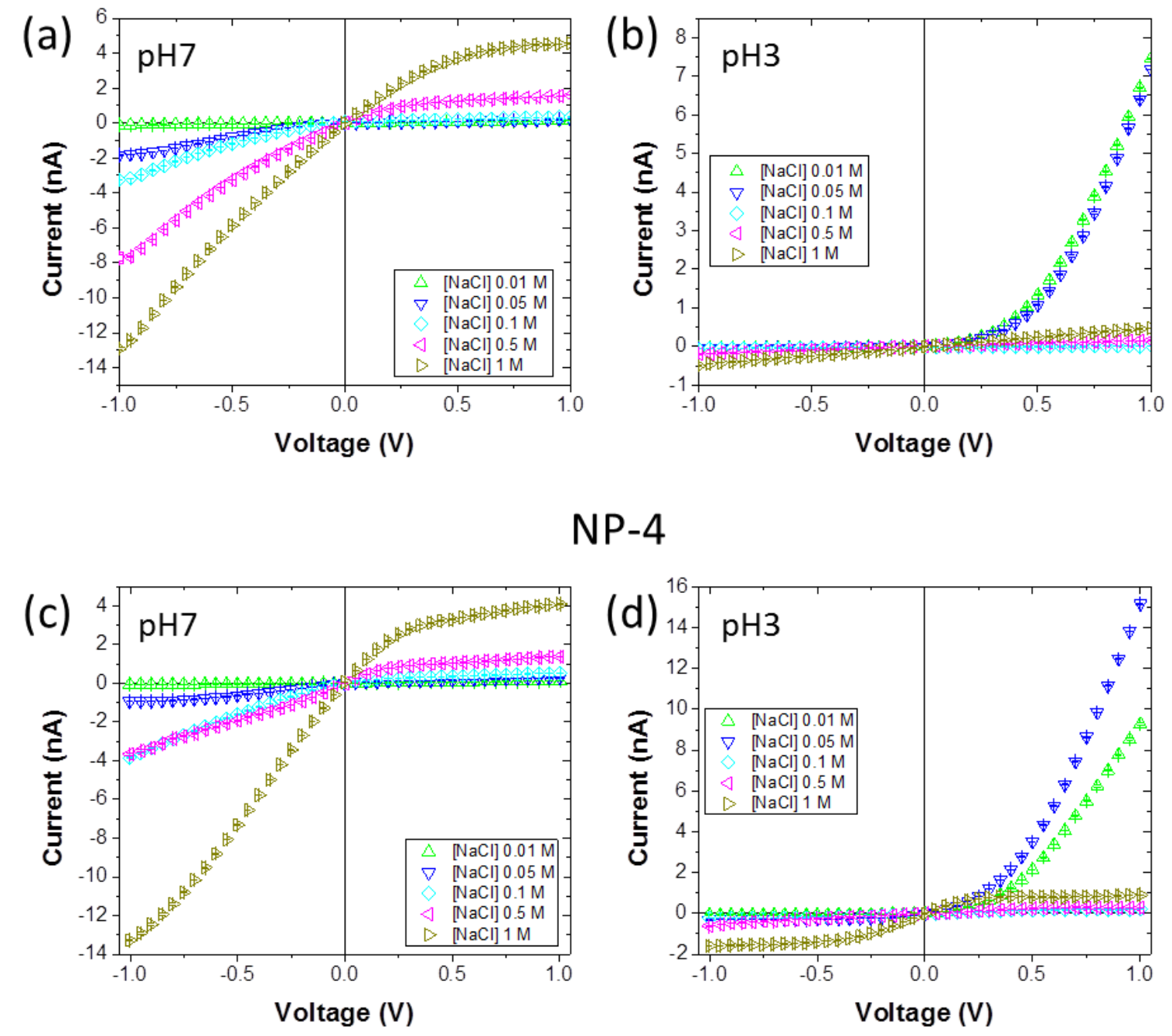

NP-4

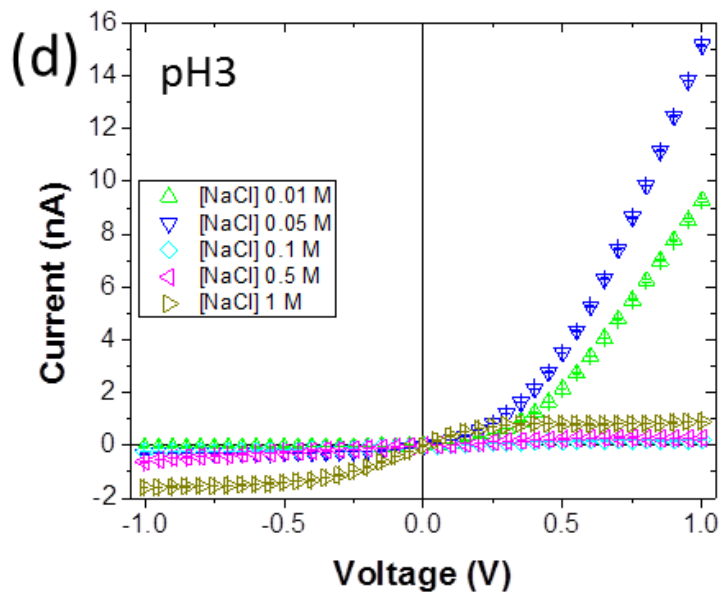

Figure 3 : I-V dependance of NP functionalized with PEI/PAA LbL at pH 7 and pH 3

To go further in our investigations, we studied the evolution of the nanopore conductance as a function of the $\mathrm{NaCl}$ concentration for different $\mathrm{pHs}$ (Figure 4). Inside a charged nanopore, the ionic conductance decreases with the salt concentration until a plateau. This constant conductivity is due to the mobile counter ions which shield the surface charge and the slippage [25-27]. At pH upper than 4, the conductance decreases with the salt concentration without reaching clearly a plateau. This result suggests a global compensation of charges between the polyations and polyanions. It is also consistent with the 
charge inversion observed after each polyelectrolyte deposition. At $\mathrm{pH} \mathrm{3,} \mathrm{the} \mathrm{conductance} \mathrm{exhibits} \mathrm{an}$ unusual dependence with the salt concentration. At concentration below $0.1 \mathrm{M}$, the conductance decreases with the salt concentration. In addition, the value of conductance is higher compared to the one obtained at greater $\mathrm{pH}$. This can be explained by the disequilibrium of charge induced by the protonation of the carboxylate groups. In order to counterbalance the excess of positive charges, $\mathrm{Cl}^{-}$ions are "pumped" inside the nanopore, increasing the concentration of mobile ions inside the nanopore. For $\mathrm{NaCl}$ concentration of $0.1 \mathrm{M}$, the conductance decreases. This nanopore enclosure could be assigned to the reorganization of the polyelectrolyte layers.

NP-2

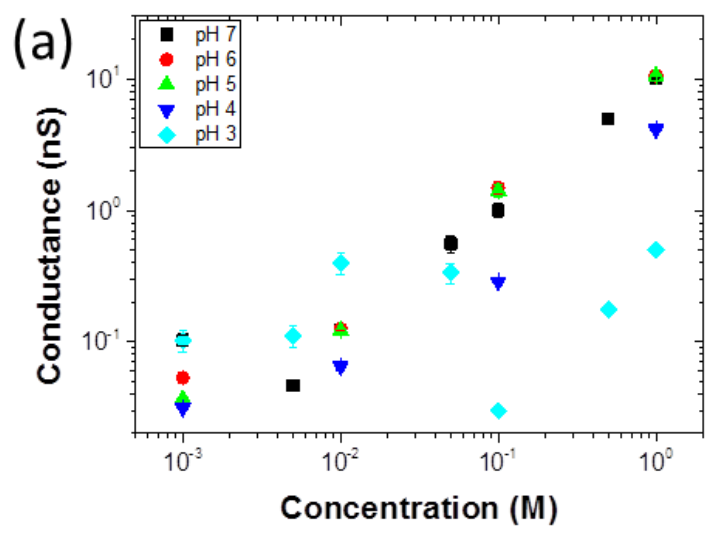

NP-4

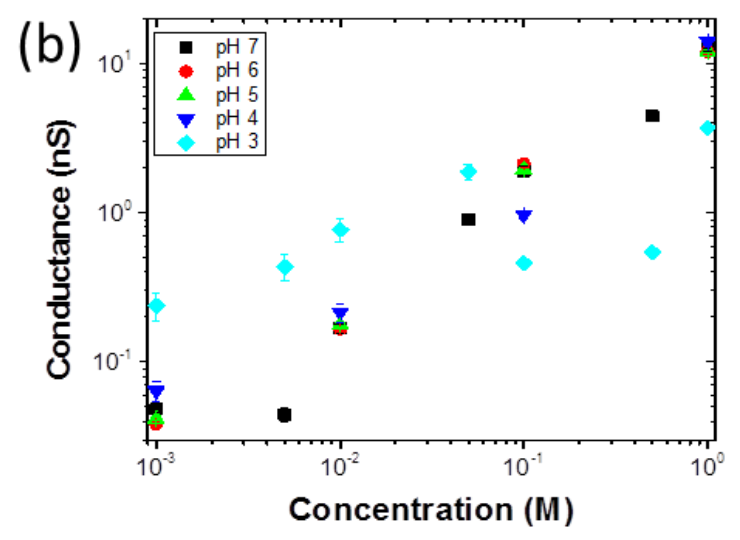

Figure 4 : dependence of the conductance vs the $\mathrm{NaCl}$ concentration of NP functionalized with PEI/PAA LbL from pH 7 to pH 3

\subsubsection{Conical nanopores decorated with PLL/PAA LbL}

The $\mathrm{NaCl}$ transport though nanopore functionalized with PLL/PAA LbL exhibits completely different behavior (Figure 5). The rectification is lower than 1 at $\mathrm{pH}$ 7. The conductance as function of the $\mathrm{NaCl}$ concentration reveals a plateau at low salt concentration as usually reported in the case of charged nanopore. The constant conductance is attributed to a partial compensation of charge between PLL and PAA. This is consistent with the fact that the PLL deposition cannot allow an inversion of rectification factor. The value of the conductance plateau is higher at $\mathrm{pH} 4$ and 3 . This can be explained by the protonation of PAA which breaks the partial balance of charge between both polyelectrolytes. In this 
case, the global charge of the polyelectrolyte layers becomes globally positive and thus we could expect an inversion of rectification factor. The I-V curves recorded at $\mathrm{pH} 3$ for two nanopores with different diameters and number of layers exhibit an opposite behavior. Indeed, at $\mathrm{pH} 3$ the rectification factor is below 1. This can be interpreted using two assumptions. The first one is the modification of PAA pKa in nanopore as predicted by theoretical model [28] and supported by previous work[29]. The second one is a modification of the polyelectrolyte conformation as it occurs for PEI/PAA or PEI/ChS [ref].
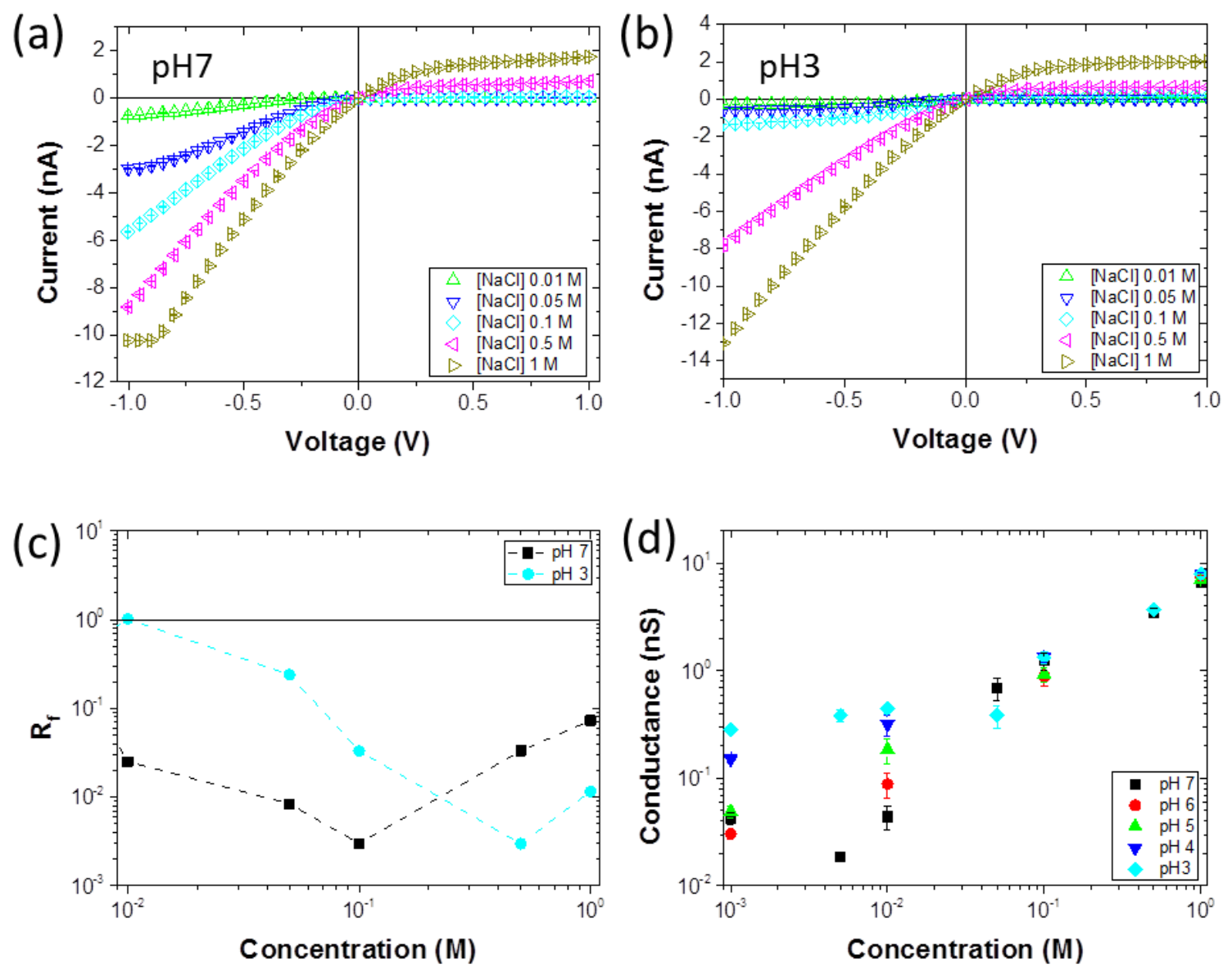

Figure 5 : I-V dependence (a-b), rectification factor (c) and conductance as a function of $\mathrm{NaCl}$ concentration (d) of NP functionalized with PEI/PAA LbL at pH 7 and $\mathrm{pH} 3$.

In order to investigate how $\mathrm{pH}$ affects the formed bilayers, we studied the impact of $\mathrm{pH}$ on PLL/PAA bilayers by total internal reflection spectroscopic ellipsometry (TIRE) (Figure 6). When increasing the $\mathrm{NaCl}$ concentration from $0.01 \mathrm{M}$ to $1 \mathrm{M}$, shift to the longer wavelength indicates a swelling of the 
bilayers. At $0.01 \mathrm{M}$ and $1 \mathrm{M}$ of $\mathrm{NaCl}$ curves are near each other at $\mathrm{pH} 3$ and 7 , the $\mathrm{pH}$ does not influence the bilayer swelling. Usually, weak polyelectrolyte layer like PAA swells at pH below their pKa [30]. According to the TIRE results, the non-inversion of the I-V dependence at $\mathrm{pH} 3$ could be attributed to a displacement of the pKa of PAA.
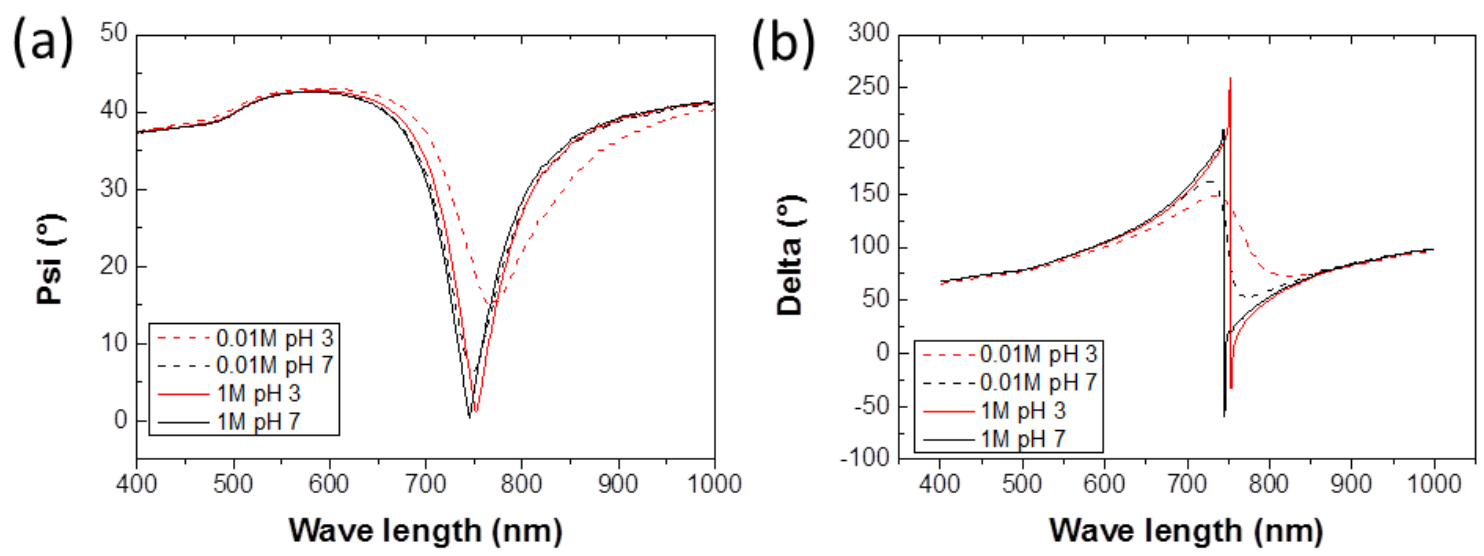

Figure 6 : Real time monitoring of ellipsometric parameters Psi and Delta using $\mathrm{pH}$ and $\mathrm{NaCl}$ concentration

\subsection{Ionic transport through nanopores functionalized by crosslinked LbL deposition}

The simple deposition of polyelectrolyte bilayer shows interesting properties as $\mathrm{pH}$ gated in the case of PEI/PAA. However, the swelling and the displacement of pKa does not make the nanopores selective to anion at low $\mathrm{pH}$ and to cation at high $\mathrm{pH}$. As previously mentioned, this goal was previously reached with chitosan/PAA. The main difference with the polycation used in this work is that chitosan is not soluble at pH upper than 5 and thus the bilayer cannot swell. In order to stabilize the polyelectrolyte layer, one solution is to crosslink the amine and carboxylic moieties after deposition. Using this strategy, we functionalized conical nanopore with PLL/PAA, after their deposition, was added at $\mathrm{pH} 8$ to ensure the crosslinking. The evidence of each step was characterized by a modification of the rectification ratio. After functionalization, the $\mathrm{I}-\mathrm{V}$ response was recorded at different $\mathrm{NaCl}$ concentrations from $10^{-3} \mathrm{M}$ to 1 $\mathrm{M}$ at $\mathrm{pH} 8$ and 3 (Figure 7). They follow the same behavior independently of the $\mathrm{NaCl}$ concentration. At 
$\mathrm{pH} 8$, the current rectification is below 1 . This mean that the nanopore enhances the transport of cation (here $\mathrm{Na}^{+}$) compared to $\mathrm{Cl}^{-}$. This is confirmed by the permeation ratio $\mathrm{P}_{\mathrm{Na}+} / \mathrm{P}_{\mathrm{Cl}-}=8$ measured under dissymmetrical condition. This result is assigned to a charge globally negative of the nanopore. This is a reasonable assumption because the last layer is polyanions, the nanopore surface is totally deprotonated. At $\mathrm{pH} 3$, an inversion of the current rectification occurs since the factor is upper than 1 . In this case, the transport of $\mathrm{Cl}^{-}$is favored compared to the $\mathrm{Na}^{+}$as confirmed by a permeation ratio $\mathrm{P}_{\mathrm{Na}+} / \mathrm{P}_{\mathrm{Cl}}=0.01$. This result is assigned to a global positive charge of the region close to the narrow opening where are located the polyelectrolyte layers. Indeed at this $\mathrm{pH}$, the PAA nanopore surface are protonated and PLL are totally protonated.
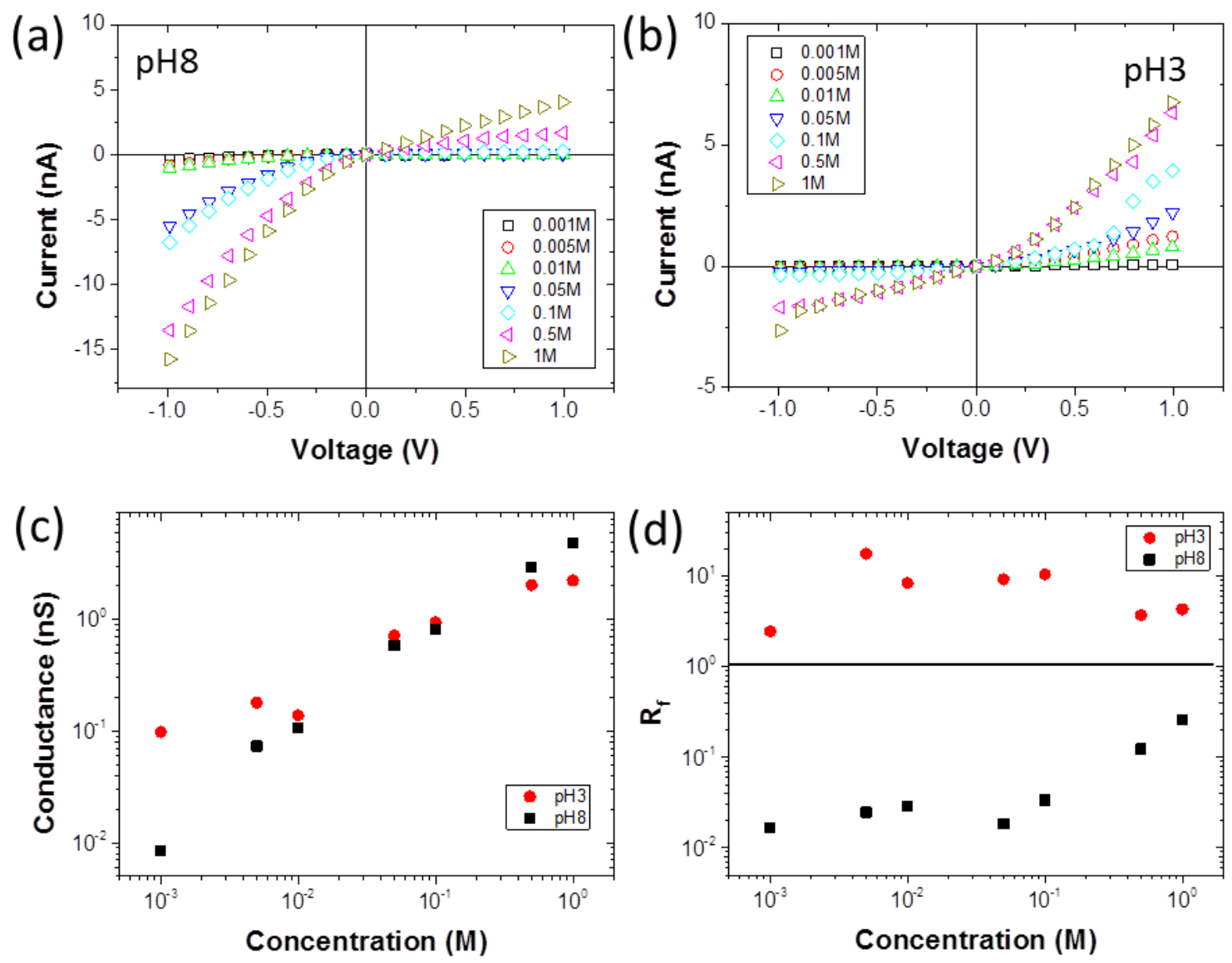

Figure $7:$ I-V dependence (a-b), rectification factor (c) and conductance as a function of $\mathrm{NaCl}$ concentration (d) of NP functionalized with PEI/PAA LbL at pH 7 and $\mathrm{pH} 3$. 
The conductance dependence with the salt concentration does not follow the same behavior at $\mathrm{pH} 3$ and $\mathrm{pH}$ 8. At $\mathrm{pH} \mathrm{3,} \mathrm{the} \mathrm{conductance} \mathrm{decreases} \mathrm{with} \mathrm{the} \mathrm{salt} \mathrm{concentration} \mathrm{until} \mathrm{reaching} \mathrm{a} \mathrm{plateau.} \mathrm{The}$ constant conductance is assigned to the counter mobile anions present inside the nanopore to shield the excess of positive charges in the polyelectrolyte layers. At $\mathrm{pH} 8$, the constant value at low concentration is not observed suggesting charge compensation between the electrolyte and eventually counter ion trapped inside layer. This interpretation seems in contradiction with the rectification below 1 suggesting a preferential transport of cation. But it has to be stressed out that the functionalization occurs only at the narrow aperture, the rest of the nanopore is negatively charged. This dissymmetry of charges along the nanopore explains the current rectification.

\section{Conclusion}

To sum up, our work aimed to fashion a nanopore which offers the possibility to drive the ionic properties by $\mathrm{pH}$. To do so, we have explored an alternative method compared to the chemical grafting of amphoteric molecule. The starting assumption was that polyelectrolyte multilayer create a zwiterionic film on nanopore surface where the charged can be modulate by the $\mathrm{pH}$. The simple deposition of PLL/PAA and PEI/PAA LbL cannot allow to validate this assumption due to the swelling of the polymer layer. However, interesting ionic transport properties were obtained for PEI/PAA because at $\mathrm{pH} 3$ the nanopore is only at low salt concentration while at $\mathrm{pH} 7$ it is already open. In order to prevent the swelling, the crosslinking of PLL/PAA was performed. As assumed in this case, the $\mathrm{pH}$ allows driving the ionic diode properties. Because, the track-etched technique allows to obtain a single pore as well as multipore, the upscale of these properties to a membrane can be considered. Beside the interest of this study to design responsive membrane, the work on a single nanopore allows a characterization of the behavior of confined polyelectrolyte layer. 


\section{ACKNOWLEDGMENT}

Single tracks have been produced in GANIL (Caen, France) in the framework of an EMIR project.

\section{References}

1. Lepoitevin, M., et al., Functionalization of single solid state nanopores to mimic biological ion channels: a review. Advances in Colloid and Interface Science, 2017.

2. Zhang, H.C., Y. Tian, and L. Jiang, Fundamental studies and practical applications of bioinspired smart solid-state nanopores and nanochannels. Nano Today, 2016. 11(1): p. 61-81.

3. Constantin, D. and Z.S. Siwy, Poisson-Nernst-Planck model of ion current rectification through a nanofluidic diode. Physical Review E, 2007. 76(4).

4. Siwy, Z., et al., Conical-nanotube ion-current rectifiers: The role of surface charge. Journal of the American Chemical Society, 2004. 126(35): p. 10850-10851.

5. Apel, P.Y., et al., Diode-like single-ion track membrane prepared by electro-stopping. Nuclear Instruments \& Methods in Physics Research Section B-Beam Interactions with Materials and Atoms, 2001. 184(3): p. 337-346.

6. Harrell, C.C., Z.S. Siwy, and C.R. Martin, Conical Nanopore Membranes: Controlling the Nanopore Shape. Small, 2006. 2(2): p. 194-198.

7. Cervera, J., B. Schiedt, and P. Ramirez, A Poisson/Nernst-Planck model for ionic transport through synthetic conical nanopores. Europhysics Letters, 2005. 71(1): p. 35-41.

8. Cervera, J., et al., Ionic conduction, rectification, and selectivity in single conical nanopores. Journal of Chemical Physics, 2006. 124(10): p. 104706.

9. Ali, M., et al., Logic Gates Using Nanofluidic Diodes Based on Conical Nanopores Functionalized with Polyprotic Acid Chains. Langmuir, 2009. 25(20): p. 11993-11997.

10. Ali, M., R. Neumann, and W. Ensinger, Sequence-Specific Recognition of DNA Oligomer Using Peptide Nucleic Acid (PNA)-Modified Synthetic Ion Channels: PNA/DNA Hybridization in Nanoconfined Environment. Acs Nano, 2010. 4(12): p. 7267-7274.

11. Lepoitevin, M., et al., Non-Fluorescence label protein sensing with track-etched nanopore decorated by avidin/biotin system. electrochemica acta, 2016. 211: p. 611-618.

12. Lepoitevin, M., et al., Combining a sensor and pH-gated Nanopore based on an Avidin-biotin system Chemical Communications, 2015. 51: p. 5994-5997

13. Zhao, Y., et al., Mimicking pH-gated ionic channel by polyelectrolyte complex confinement inside single nanopore. Langmuir, 2017. 33: p. 3484-3490.

14. Ma, T., et al., Combining Light-Gated and pH-responsive nanopore based on PEG-spiropyran functionalization Advanced Materials Interfaces, 2017.

15. Zhang, M.H., et al., Light and pH Cooperative Nanofluidic Diode Using a SpiropyranFunctionalized Single Nanochannel. Advanced Materials, 2012. 24(18): p. 2424-2428.

16. Ali, M., et al., Biosensing and Supramolecular Bioconjugation in Single Conical Polymer Nanochannels. Facile Incorporation of Biorecognition Elements into Nanoconfined Geometries. Journal of the American Chemical Society, 2008. 130(48): p. 16351-16357.

17. Ali, M., et al., Label-free histamine detection with nanofluidic diodes through metal ion displacement mechanism. Colloids and Surfaces B: Biointerfaces, 2017. 150: p. 201-208.

18. Yameen, B., et al., Proton-regulated rectified ionic transport through solid-state conical nanopores modified with phosphate-bearing polymer brushes. Chemical Communications, 2010. 46(11): p. 1908-1910. 
19. Ali, M., et al., A pH-Tunable Nanofluidic Diode with a Broad Range of Rectifying Properties. Acs Nano, 2009. 3(3): p. 603-608.

20. Alem, H., et al., Layer-by-layer assembly of polyelectrolytes in nanopores. Macromolecules, 2007. 40(9): p. 3366-3372.

21. Lepoitevin, M., et al., Fast and reversible functionalization of a single nanopore based on layerby-layer polyelectrolyte self-assembly for tuning the current rectification and designing sensors. RSC Advances, 2016. 6: p. 32228.

22. Ali, M., et al., Layer-by-Layer Assembly of Polyelectrolytes into Ionic Current Rectifying SolidState Nanopores: Insights from Theory and Experiment. Journal of the American Chemical Society, 2010. 132(24): p. 8338-8348.

23. Balme, S., et al., Large osmotic energy harvesting from functionalized conical nanopore suitable for membrane applications Journal of Membrane Science, 2017. 544: p. 18-24.

24. Harrell, C.C., S.B. Lee, and C.R. Martin, Synthetic single-nanopore and nanotube membranes. Analytical Chemistry, 2003. 75(24): p. 6861-6867.

25. Balme, S., et al., Ionic transport through sub $10 \mathrm{~nm}$ hybrophobic nanopore : experiment, theory and simulation. scientific reports, 2015. 5: p. 10135.

26. Secchi, E., et al., Massive radius-dependent flow slippage in carbon nanotubes. Nature, 2016. 537(7619): p. 210-213.

27. Siria, A., et al., Giant osmotic energy conversion measured in a single transmembrane boron nitride nanotube. Nature, 2013. 494(7438): p. 455-458.

28. Tagliazucchi, M., O. Azzaroni, and I. Szleifer, Responsive Polymers End-Tethered in Solid-State Nanochannels: When Nanoconfinement Really Matters. Journal of the American Chemical Society, 2010. 132(35): p. 12404-12411.

29. Yameen, B., et al., Single Conical Nanopores Displaying pH-Tunable Rectifying Characteristics. Manipulating Ionic Transport With Zwitterionic Polymer Brushes. Journal of the American Chemical Society, 2009. 131(6): p. 2070-2071.

30. Shiratori, S.S. and M.F. Rubner, pH-dependent thickness behavior of sequentially adsorbed layers of weak polyelectrolytes. Macromolecules, 2000. 33(11): p. 4213-4219. 


\section{Supporting information: Design of pH-responsive nanopore using polyelectrolyte confinement.}

Tianji Ma, Paulius Gaigalas, Mathilde Lepoitevin, Ieva Baleviciute-Plikusiene, Mikhael Bechelany, JeanMarc Janot, Emmanuel Balanzat, and Sebastien Balme

SI-1 Characterization of polyelectrolyte growth by spectroscopic ellipsometry
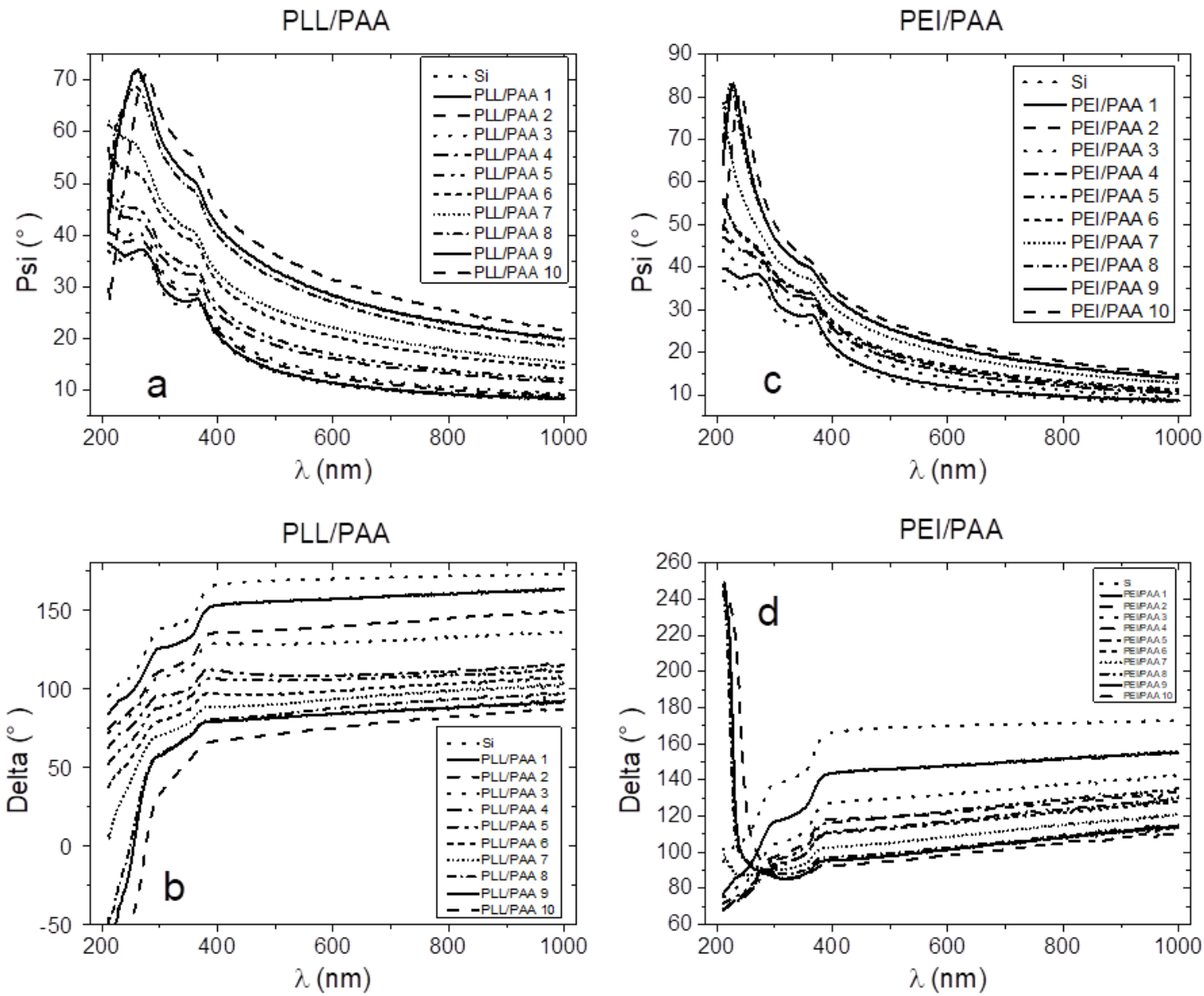
Figure SI-1a. a and b ellipsometric parameters Psi and Delta dependence on wavelength respectively for each additionally grown PLL/PAA layer, $\mathrm{c}$ and d ellipsometric parameters Psi and Delta dependence on wavelength respectively for each additionally grown PEI/PAA on the silicon substrate surface.

In order to investigate the kinetics of PLL/PAA layers formation, the total internal reflection spectroscopic ellipsometry was used. For this purpose custom made liquid cell for in-situ kinetics investigation was used together with attached $70^{\circ} \mathrm{BK} 7$ glass prism coated with a gold plate for surface plasmon resonance excitation in order to have a better sensitivity [In situ study of ligand-receptor interaction by total internal reflection ellipsometry, Z Balevicius, I Baleviciute, S Tumenas, L Tamosaitis, A Stirke, A Makaraviciute, A Ramanaviciene, A Ramanavicius, 2014/11/28, Thin Solid Films, 571, 744748]. 10 PLL/PAA bilayers were grown onto the gold surface. After each additional layer was grown the cell was washed with deionized water. The shift of Psi and Delta curves after each washing step are presented in figure XXX a, b, c, d . The shift to longer wavelength at different time moments indicate that each additional layer is formed on the top of previously formed layer and after washing new layer remains on the surface.
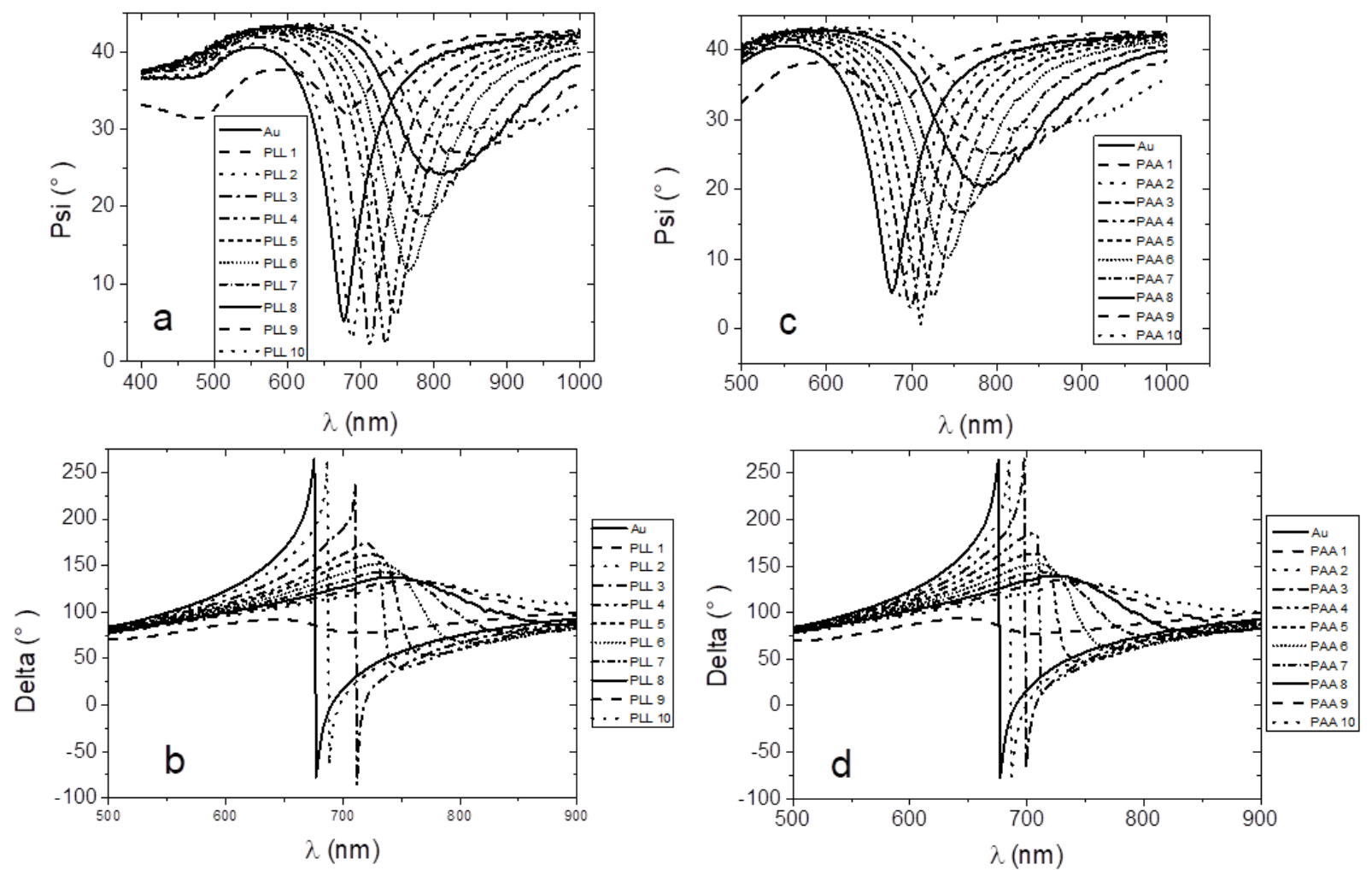
Figure SI-1b. Real time monitoring of ellipsometric parameters Psi and Delta for PLL (a, b) and for PAA (c, d) layers on gold plate.
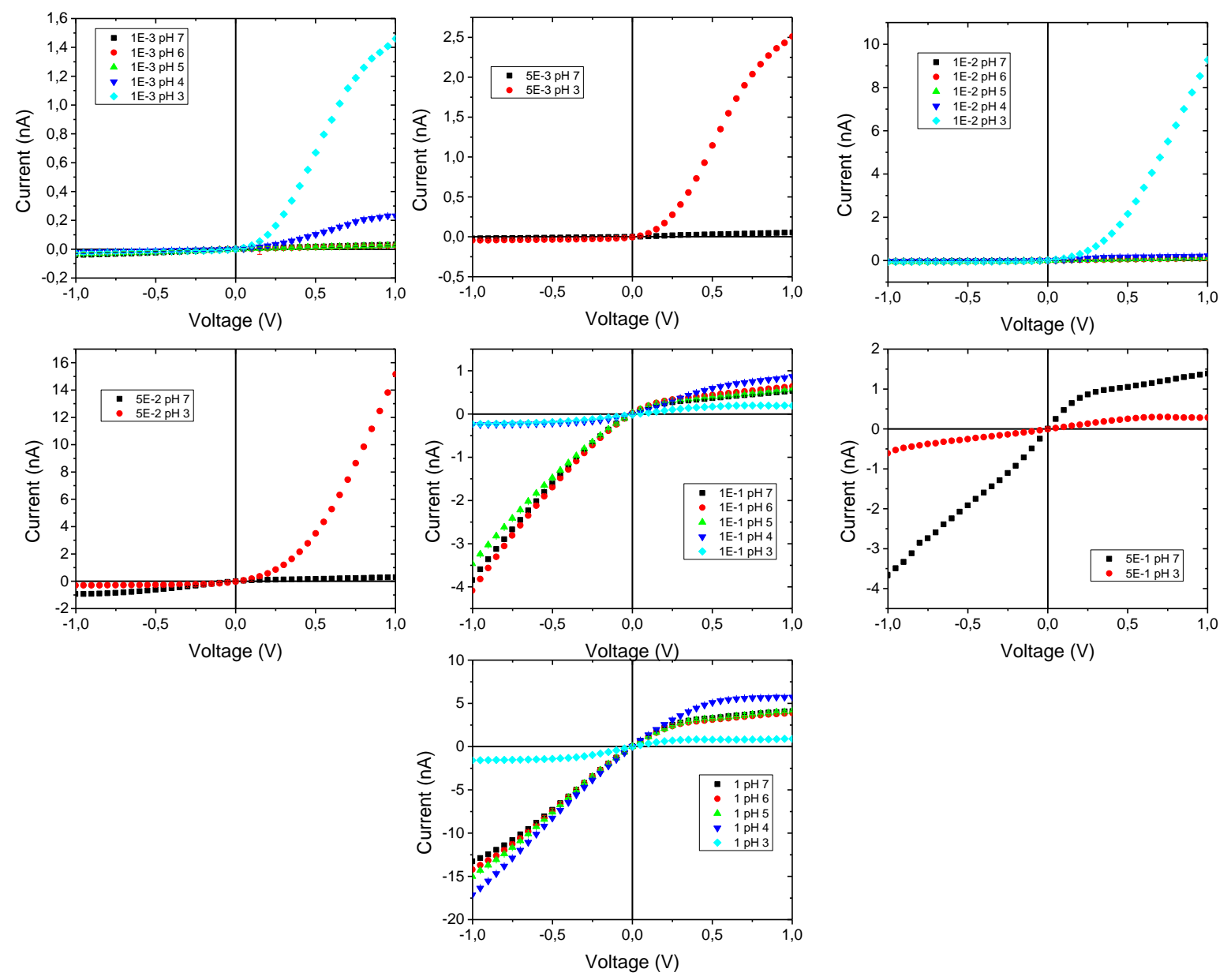

Figure SI-2 : I-V dependence of nanopore $(\mathrm{d}=98 \mathrm{~nm})$ functionalized with PEI/PAA 10 bilayers 


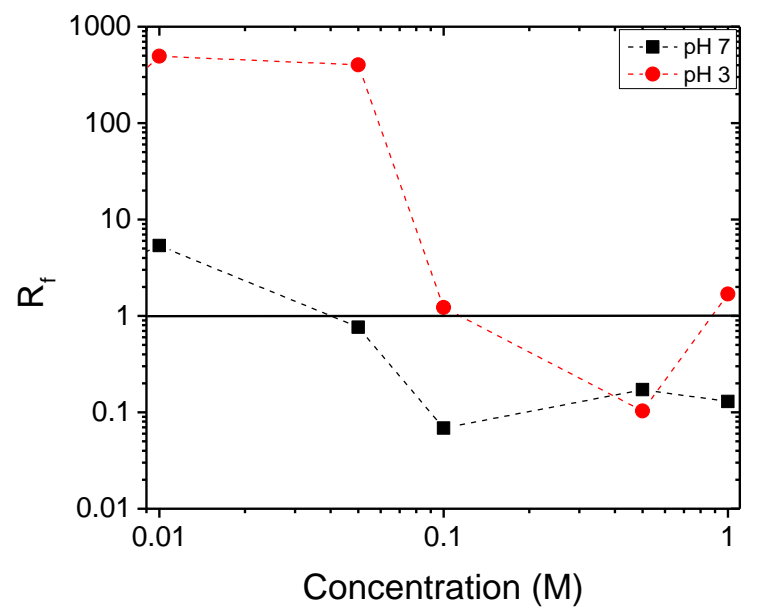

Figure SI-3 : rectification factor of nanopore $(\mathrm{d}=98 \mathrm{~nm})$ functionalized with PEI/PAA 10 bilayers
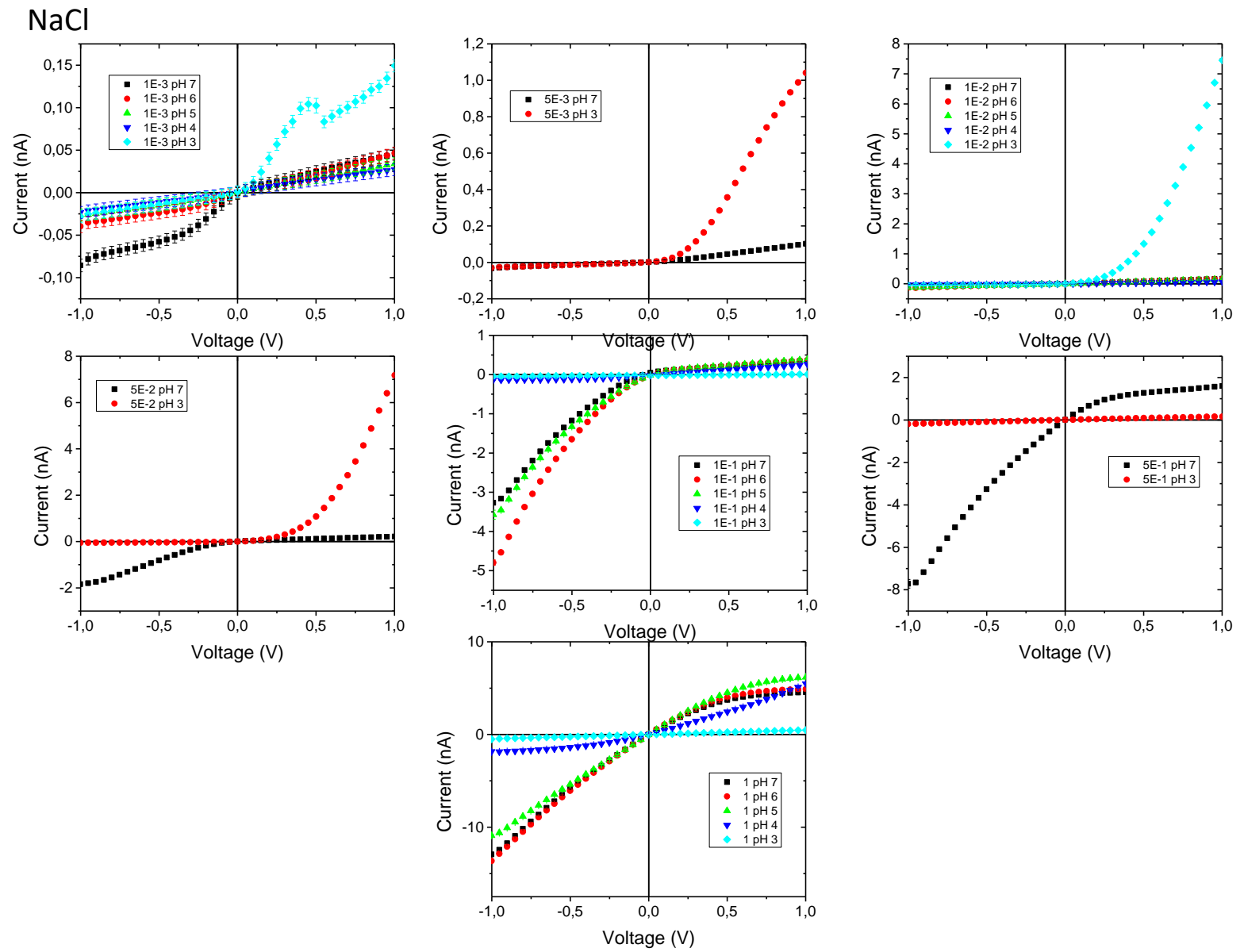

Figure SI-4 : I-V dependence of nanopore $(\mathrm{d}=28 \mathrm{~nm})$ functionalized with PEI/PAA 4 bilayers 

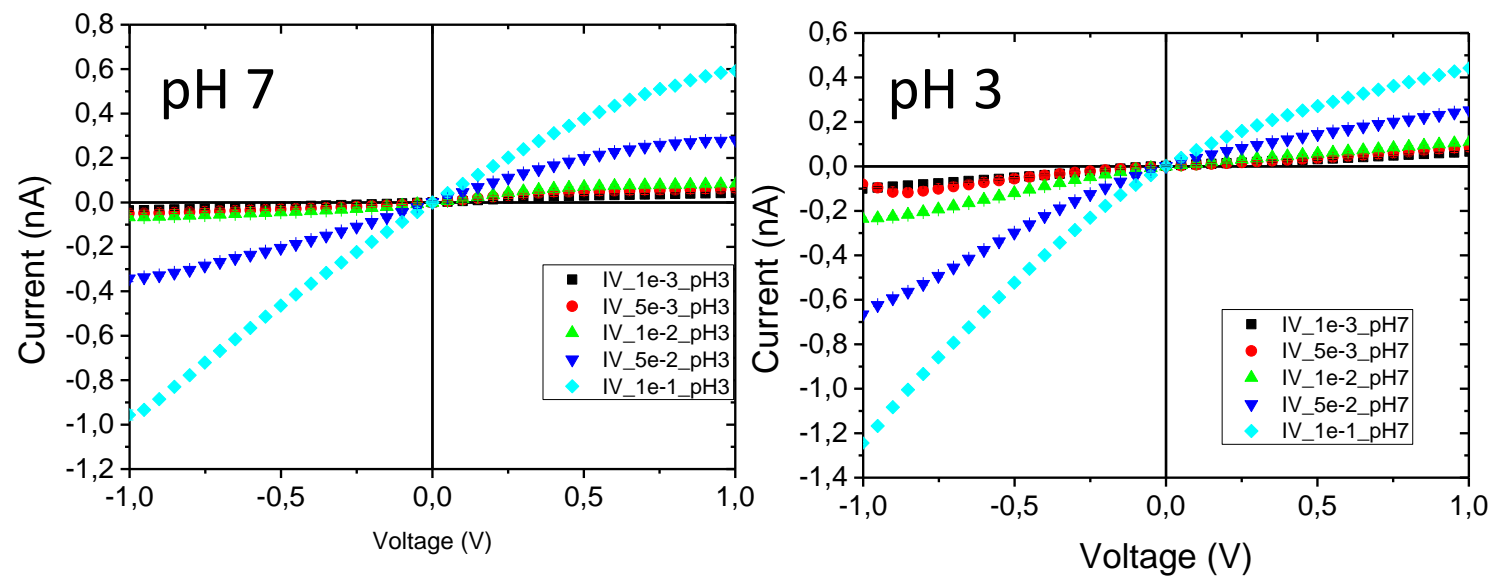

Figure SI-5 : I-V dependence of nanopore $(\mathrm{d}=28 \mathrm{~nm})$ functionalized with PLL/PAA 4 bilayers 